\title{
Multi-modality Therapies for Advanced Small Cell Carcinoma of the Ovary, Hypercalcemic Type (SCCOHT): Three Case Reports and Literature Review
}

Qin Han ( $\sim$ Lmlfe_lynn@126.com )

The Third Hospital of Peking University

Hongyan Guo

The Third Hospital of Peking University

\section{Case report}

Keywords: small cell carcinoma of the ovary hypercalcemic type, SCCOHT, advanced stage, tumor immunotargeted therapy

Posted Date: March 22nd, 2021

DOl: https://doi.org/10.21203/rs.3.rs-320903/v1

License: (c) (i) This work is licensed under a Creative Commons Attribution 4.0 International License. Read Full License

Version of Record: A version of this preprint was published at European Journal of Gynaecological Oncology on January 12th, 2022. See the published version at https://doi.org/10.31083/j.ejgo4301002. 


\section{Abstract}

\section{Background}

Small-cell carcinoma of the ovary, hypercalcemic type (SCCOHT) is a rare but highly undifferentiated, aggressive malignancy that primarily affects young women. Due to the early onset, unclear familial history, and vague presenting symptoms, most SCCOHT patients present with advanced disease and have a very poor prognosis. Although several therapeutic regimens have been proposed, there is no consensus on the optimal strategy.

Case presentation

We described three cases of advanced-stage SCCOHT treated with cytoreductive surgery and chemotherapy with or without immune checkpoint blockade treatment. The patients showed different survival rates.

\section{Conclusions}

Given the almost universal mortality of advanced SCCOHT during long-term follow-up, we believe these cases highlight the importance of prompt diagnosis as well as early, aggressive, and combined modality treatment of SCCOHT. We also believe that improved therapies could result in more young patients surviving SCCOHT.

\section{Background}

Small-cell carcinoma of the ovary, hypercalcemic type (SCCOHT), is a rare, highly undifferentiated, aggressive ovarian malignancy with an unknown cell of origin, which usually occurs in young women. It was first described by Dickersin et a/ in 1982 as paraneoplastic hypercalcemia[1]. Several therapeutic regimens have been proposed for SCCOHT; however, to date, there is no consensus on the optimal strategy. With the development of high-throughput sequencing technology, molecular characteristics of SCCOHT have been gradually revealed, and new molecular targets have been proposed. Herein, we described three SCCOHT cases with different clinical stages, different treatment, and different outcomes and presented an overview of the clinical and pathological features, molecular features, multi-mode therapy, molecular targeting and immunotherapy, genetic counseling, and prevention of SCCOHT.

\section{Case Presentation}

\section{Case A}

Patient A was a 36-year-old G2P1 female who was admitted to the Third Hospital of Peking University in December 2019 with acute abdominal pain, and abdominal distension accompanied by fever. Bilanual gynecological examination revealed a $9 \mathrm{~cm}$ cystic, solid mass in front of the uterus. Serum calcium and 
tumor markers were within normal limits. Pelvic abdominal computerized tomography (CT) indicated a $9.6 \times 9.2 \mathrm{~cm}$ mass extending from the right ovary (Figure 1). Emergent laparoscopy exploration was performed. The right ovary had a solid but brittle enlargement of $10 \mathrm{~cm}$ with a rich blood supply (Figure 1). In addition, a resection of the right adnexa was performed. The frozen section analysis suggested a poorly differentiated carcinoma. As metastatic cancer was not excluded, only the right adnexa was removed. The pathological results of paraffin reported small-cell carcinoma of the ovary, hypercalcemic type (SCCOHT) (Figure 1).

Considered that the operation was just performed, and the pelvic area was in acute inflammatory and hyperemia edema period, she received three courses of chemotherapy of TC (paclitaxel $135-175 \mathrm{mg} / \mathrm{m} 2$ d1 and carboplatin AUC 5-6 d1, intravenous infusion) between January and March 2020. The regimen was given every 21 days. During the cytoreductive surgery in April 2020, we found several 1-2cm masses on the surface of the left fallopian tube and appendix. The tumor was classified as stage IIIB. Next, a genetic test was performed by LIFE HEALTHCARE, showing a mutation of SMARCA4; germline testing was negative. The score of HRD and PD-L1 (TPS) was 25 and 10-15\%, respectively. According to the guidelines published by the International SCCOHT Consortium in March 2020, six courses of EP regimen (etoposide $100 \mathrm{mg} / \mathrm{m} 2 \mathrm{~d} 1$ to $\mathrm{d} 3$, cis-platinum $70 \mathrm{mg} / \mathrm{m} 2 \mathrm{~d} 1$, intravenous infusion) were given postoperation. The regimen was given every 21 days.

In August 2020, a $1 \mathrm{~cm}$ mass in the right lower abdominal wall was found on the CT, just after the last chemotherapy cycle. The pathology suggested a metastatic lesion of SCCOHT. Considering the disease was not controlled during both the preoperative TC chemotherapy and postoperative EP chemotherapy, cancer-targeted drugs were prescribed. Five courses of PD1 (sintilimab 200mg injection, the regimen was given for every 21 days.) were given between August and December 2020. Ponatinib (protein receptor tyrosine kinase inhibitor) $45 \mathrm{mg}$ qd was used as a maintenance treatment. Follow-up was performed every three months (laboratory tests and ultrasound). At the last follow-up (January 2021), the patient was still alive, showing no recurrence signs.

\section{Case B}

Patient B was a 15 years old girl who was admitted to The Third Hospital of Peking University emergency department in October 2012 due to epigastric pain and a high fever $\left(38^{\circ} \mathrm{Cp}\right.$. Physical examination revealed under xiphoid process and left abdominal tenderness and a large pelvic mass. Pelvic abdominal CT suggested a $25 \mathrm{~cm}$ cystic mass; thus, malignancy was suspected (Fig. 2). The CA125 was high at $345.7 \mathrm{U} / \mathrm{mL}(0-35 \mathrm{U} / \mathrm{ml})$. An emergent open exploratory operation was performed, during which a large cystic, solid mass from the left ovary was found in the whole pelvic cavity. Multiple peritoneal nodules were found in the pelvic cavity. The frozen section analysis suggested a poorly differentiated carcinoma. Cytoreductive surgery was performed immediately after the diagnosis was confirmed and after the signed informed content from the patient's parents was obtained. We performed a successful cytoreductive surgery (R1) with a $1 \mathrm{~cm}$ mass left in the cavity between liver, stomach, and pancreas, followed by an intraperitoneal chemoperfusion of $60 \mathrm{mg}$ cis-platinum ten days after the surgery. The pathological results of paraffin reported SCCOHT and were classified as stage IIIC. 
Fourteen days after the operation, an abdominal ultrasound indicated a $7 \mathrm{~cm}$ nodule between the liver, stomach, and pancreas, which was residual lesion growth, and a $1 \mathrm{~cm}$ lesion on the liver surface, which was considered a metastatic lesion. Six courses of EP regimen (etoposide $100 \mathrm{mg} / \mathrm{m} 2 \mathrm{~d} 1$ to d3, cisplatinum $70 \mathrm{mg} / \mathrm{m} 2 \mathrm{~d} 1$, intravenous infusion) were given post-operation. The regimen was given every 21 days. Unfortunately, the abdominal mass increased in size (to $9 \mathrm{~cm}$ )(Fig. 2). Her parents refused further treatment, and the patient passed away due to multiple organ failure in July 2017. The survival time was nine months after diagnosis.

\section{Case C}

Patient $\mathrm{C}$ was a 33 years old girl who suffered from abdominal distension for two months. She was diagnosed with myoma in uterus and underwent laparotomy hysterectomy surgery in another hospital in November 2018. The pathological results of paraffin of the mass initially revealed a high-grade serous carcinoma of the ovary. Based on this diagnosis, the patient received three-course chemotherapy of TC (paclitaxel 135-175mg/m2 d1and carboplatin AUC 5-6 d1, intravenous infusion). Unfortunately, a rectovaginal fistula was found after the first course, and the tumor mass increased during the chemotherapy with metastasis of peritoneum omentum and vaginal cuff. In February 2019, after pathology consultation in The Third Hospital of Peking University, she was diagnosed with SCCOHT, stage IVb. Considering the progression, immunity inhibitors and anti-angiogenesis were used. Three courses of chemotherapy with albumin taxol, cis-platinum, bevacizumab and PD1 (albumin-bound paclitaxel 135-175mg/m2 d1, cis-platinum 70-75mg/m2 d1, bevacizumab 15mg/kg d2, sintilimab 180mg $\mathrm{d} 3$, intravenous infusion)) were given before the second surgery. The regimen was given every 21 days. The tumor mass was reduced during chemotherapy. The second cytoreductive surgery was performed in June 2019 , during which a $5 \mathrm{~cm}$ mass was identified on the vaginal stump. Luckily, the mass was removed without an enterostomy. The second cytoreductive surgery was R0. Five courses of chemotherapy of albumin-bound paclitaxel, cis-platinum, and PD1 were performed after the surgery. Currently, the patient has been taking PD1 as maintenance treatment for the past 2 years. Follow-up was performed every three months (laboratory tests and ultrasound). At the last follow-up (January 2021), the patient was still alive with no signs of recurrence.

\section{Discussion And Conclusions}

\section{Clinical and pathology features}

The SCCOHT is a rare ovarian tumor with poor prognosis that was first described in 1982[1]. In the landmark study by Young et al [2], the clinical and pathological features of SCCOHT were analyzed. The minimum age of onset was 14 months. The tumor primarily affected young women with a median of 23 years. The clinical signs were nonspecific and mainly included abdominopelvic pain or distension, weight loss, nausea, and vomiting; approximately $60 \%$ of SCCOHT cases presented with paraneoplastic hypercalcemia[2, 3]. The recurrence rate was $65.1 \%$, and the average recurrence time was 11.5 months[4, 5]. The three cases discussed in this report were all PD (progression disease) after the initial treatment. 
Most patients are diagnosed at an advanced stage, and the overall long-term survival rate in SCCOHT patients is only $10-20 \%[4,6,7]$.

The tissue origin of SCCOHT has been inconclusive. A small cluster of round or oval cells with sparse cytoplasm, hyperchromatic nuclei, prominent nucleoli, and active mitotic figures was found in all three cases presented in this study (Fig. 3). The pathological basis of SCCOHT still remains unclear but suggests a primitive germ cell origin. The research found that SMARCA 4 (SWI/SNF related, matrix associated, actin-dependent regulator of chromatin, subfamily member 4) gene mutation and the relative BRG-1 protein deficiency is the important molecular feature of SCCOHT (Fig. 3) [8, 9].

\section{Clinical management}

To date, there is no consensus on the optimal strategy for SCCOHT. Among three cases presented in this study, patient $B$ had the earliest occurrence time and the worst prognosis, which may be related to the lack of experience in the diagnosis and treatment of this rare disease at that time. The development of pathogenesis and related targeted drugs has gradually brought certain benefits to patients. Also, the International SCCOHT Consortium (ISC) presented guidelines for the diagnosis and management of SCCOHT in 2020[10].

For newly diagnosed disease, primary ovarian tumor resection and diagnosis by gynecologic pathology are necessary. In this report, cytoreductive surgery was performed in all three cases. Initiate cytotoxic chemotherapy included cisplatin and etoposide regimens (e.g., BEP or VPCBAE or similar). For patient A, three courses of TC as neoadjuvant chemotherapy were used. As the disease progressed and tumor mass was found during the second surgery, chemotherapy was changed to EP. Radiation therapy may be considered after chemotherapy for the residual disease, but the efficacy remains uncertain.

For recurrent disease, the principles for recurrent ovarian cancer are applicable. As chemotherapy has limited effectiveness, surgery can be given after the administration of targeted drugs and immunotherapy. Chemotherapy with cisplatin and etoposide regimens or alternative chemotherapy regimens (cyclophosphamide, doxorubicin, vincristine; or carboplatin, paclitaxel; or topotecan; or similar) can be combined with other treatments. SCCOHT patients should be encouraged to participate in clinical trials if available and consider off-label immune checkpoint blockade treatment based on drug availability. Patient A used PD1 and ponatinib; Patient C used PD1 and Bevacizumab. Both patients benefited from the off-label drugs, which will be discussed more in detail.

\section{Identification of sccoht therapeutic candidates}

SMARCA4 is involved in many cellular processes, including transcriptional regulation, DNA damage repair, differentiation, and mitosis, all of which may contribute to SCCOHT phenotypes. The targeted drug should focused on several approaches, including exploiting known synthetic lethal interactions of SMARCA4 loss and identifying novel targets through unbiased genetic screens. 


\section{Epigenetic therapeutics (EZH2 inhibitor, LSD1 inhibitor, BET inhibitor, lysine-specific demethylase inhibitor)}

Impaired function of the mSWI/SNF complex leads to histone-lysine N-methyltransferase (EZH2) abnormal recruitment to target genes[11]. Selective EZH2 inhibitor tazemetostat has shown an anti-tumor effect in a rat model. Recently, tazemetostat has been approved by the FDA for the treatment of epithelioid sarcoma. The arm accruing patients with SCCOHT in phase II multiarm trial (ClinicalTrials.gov identifier: NCT02601950) was stopped for futility. Of note, 1 patient with SCCOHT was reported to have a PR of 32 weeks $[12,13]$. One way to improve efficacy may be to combine EZH2 inhibitors with histone deacetylase inhibitors, such as panobinostat or quisinostat, which demonstrated a greater synergistic anti-tumor effect than monotherapy[12].

A similar approach is used with inhibitors of lysine-specific demethylase-1 (LSD1/KDM1), which is upregulated in SWI/SNF-mutated cancers. The LSD1 inhibitor seclidemstat (SP-2577) demonstrated antitumor activity in murine models. A phase I trial (NCT03895684) has been recently designed to investigate the clinical effect of seclidemstat, for patients with SWI/SNF-mutant gynecologic cancers, with an emphasis on SCCOHT, ovarian clear cell carcinomas, and endometrial carcinomas.

Bromodomain and extraterminal motif (BET) inhibitors are other potential drugs for SCCOHT. In vivo studies showed anti-proliferative effects of BET inhibition such as OTX015 in SMARCA4-deficient SCCOHT by downregulating the oncogenic HER3 and other receptor tyrosine kinases[14]. BET inhibitors can also promote an anti-tumor response, decrease regulatory T cells, increase TILs, and synergism in vivo with anti-PD1 therapy[15].

Targeting other histone modification complexes, HDACi has been clinically approved for the treatment of several hematologic malignancies. Several studies have shown that HDAC is in the context of SCCOHT results in re-expression of SMARCA2, which strongly suppresses the growth of SCCOHT cells[16]. One of these reports also showed in vivo sensitivity of SCCOHT cells to the HDACi Quisinostat.

\section{Kinase inhibitors (RTK inhibitor, CDK4/6 inhibitor, c-Met inhibitor)}

A previous study showed the SCCOHT cell line's dependence on a variety of protein receptor tyrosine kinase (RTK)[17]. Ponatinib, an RTK inhibitor approved for clinical use, was found to have a strong inhibitory effect on SCCOHT cell lines. Furthermore, inhibition of EGFR2, CSF1R, and VEGFR negatively affect the recruitment and survival of myeloid-derived suppressor cells, thus suggesting ponatinib has a role in modulating the immune microenvironment for anti-tumor effect[18].

Xue et al [19] found that silencing cyclin-dependent kinase4/6 (CDK4/6) may significantly inhibit SCCOHT cell growth by screening short hairpin RNA (shRNA) library. In vitro and in vivo studies suggested that SCCOHT is sensitive to palbociclib, a CDK4/6 inhibitor that has been used in the clinical treatment of ER + and HER2-breast cancer. The mechanism of action is related to the decrease of cyclin 
D1 expression and the restriction of CDK4/ 6 kinase activity due to SMARCA4 deficiency. The Canadian Profiling and Targeted Agent Utilization Trial (NCT03297606), a pan-Canadian phase II basket trial, has recently approved a new match to treat SMARCA4-mutant tumors with the CDK4/6 inhibitor palbociclib based on the findings[19, 20].

\section{Immunotherapies (PD-1/PD-L1 inhibitor)}

Although the low mutation burden of SCCOHT would not predict responsiveness to immune checkpoint blockade (ICB), programmed cell death protein 1 (PD-1) inhibitors including pembrolizumab have shown substantial and durable responses in selected patients with recurrent SCCOHT after prior treatment[21]. A French phase II basket trial AcSe program with pembrolizumab (NCT03012620) is currently open for women with rare ovarian tumors, including relapsed SCCOHT. The application of SCCOHT's immune microenvironment and PD-1/PD-L1 inhibitor needs further study.

\section{Off-label drug discussion for the cases}

To the best of our knowledge, this is the first study reporting on the use of ponatinib or combine antiangiogenic therapy bevacizumab and immune checkpoint blockade (ICB) in patients with SCCOHT. Although patient $\mathrm{C}$ was misdiagnosed at another hospital and suffered from rectovaginal fistula even progressed during chemotherapy, after full communication with the patient, she was treated with ICB and Bevacizumab, which put the progression of the disease under control, and the second tumor cell reduction was satisfactorily performed. Currently, ICB is performed as maintenance therapy. She is among rare stage IV patients who benefited from the target drugs and was still having a good quality of life. Although patient A was timely diagnosed and received standard treatment, despite the change of chemotherapy regimen, the relapse still occurred over a short time, which might be related to the laparoscopic surgery. Considering an uncontrolled condition, five courses of ICB were performed, and Ponatinib was used as a follow-up treatment, as was also reported in previous studies. Although there are some side effects such as low fever, body aches, and leukopenia, the patients still benefited from the targeted drugs.

As more clinical trials become available for patients with SCCOHT, we suggest that immunotherapy should be the first choice of treatment if available after initial therapy. Although the first choice of drug and the course is still being explored, the use of available drugs is more important than standard care. We hope that additional viable options will become available as more preclinical and translational studies are being reported and that more SCCOHT patient will benefit from them.

\section{Genetic counseling}

SMARCA4 germline variation is still possible in SCCOHT patients who do not have a family history of SCCOHT[5]. Therefore, patients diagnosed with SCCOHT, with or without a family history of SCCOHT, are advised to seek professional genetic counseling. If SCCOHT patients do not carry the SMARCA4 germline variant, unaffected ovaries' retention may be considered based on clinical conditions, tumor stage, and other factors. All patient relatives with germline mutations should undergo genetic counseling and 
mutation detection. Considering the early onset of SCCOHT, poor prognosis, and lack of an effective test, young women with SMARCA4 germline variant, may be considered for prophylactic ovariectomy. In order to achieve fertility preservation and genetic blocking of hereditary tumor families, the carriers may be considered for assisted reproductive technologies before prophylactic ovariectomy[22].

In conclusion, the SCCOHT is a rare disease with a poor prognosis. Due to the rarity and lack of large series evaluating therapeutic strategies, the clinical diagnosis and treatment experience are worth summarizing as its molecular characteristics are likely to guide the best prevention and treatment. To achieve the most reasonable treatment and genetic tumor prevention, attention should be paid to multidisciplinary cooperation, including gynecological oncology, pathology, genetic counseling, and assisted reproduction. One of the most important things is to pay attention to the mechanism of SMARCA4 driving tumor formation and the development of targeted drugs.

Table 1

Potential treatments and trials for SCCOHT.

\begin{tabular}{|llll|}
\hline Class & Target & Drug & Clinical trial available for SCCOHT \\
\hline Epigenetic & EZH2 & Tazemetostat & Phase II NCT02601950 \\
\hline therapeutics & LSD1 & Seclidemstat & Phase I, NCT03895684 \\
\hline & HDAC & Quisinostat & \\
\hline Kinase inhibitors & RTK & Ponatinib & \\
\hline CDK4/6 & Palbociclib & Phase II, NCT03297606 \\
\hline Immunotherapies & PD-1 & Pembrolizumab & Phase II, NCT03012620 \\
\hline
\end{tabular}

\section{Declarations}

Ethics approval and consent to participate

Not applicable.

\section{Consent for publication}

All patients have consented to the submission of the case report.

\section{Availability of data and materials}

All data generated or analysed during this study are included in this published article and its supplementary material. 
The authors have no conflicts of interest to disclose.

\section{Funding}

None.

\section{Authors' contributions}

Hongyan Guo jointly conceived and directed this work. Qin Han is the leading corresponding author and had full access to all data in the study and take responsibility for the integrity of the data and the accuracy of data analysis. All authors participated in the acquisition, analysis, or interpretation of the data. Qin Han wrote the report and was involved in manuscript editing. The final version had been reviewed and approved by all authors.

\section{Acknowledgements}

The authors would like to thank all study participants who were enrolled in this study.

\section{References}

1. Dickersin GR, Kline IW, Scully RE. Small cell carcinoma of the ovary with hypercalcemia: a report of eleven cases. Cancer. 1982; 49:188-97.

2. Young RH, Oliva E, Scully RE. Small cell carcinoma of the ovary, hypercalcemic type. A clinicopathological analysis of 150 cases. The American journal of surgical pathology. 1994; 18:1102-16.

3. Matias-Guiu X, Prat J, Young RH, Capen CC, Rosol TJ, Delellis RA, Scully RE. Human parathyroid hormone-related protein in ovarian small cell carcinoma. An immunohistochemical study. Cancer. 1994; 73:1878-81.

4. Estel R, Hackethal A, Kalder M, Münstedt K. Small cell carcinoma of the ovary of the hypercalcaemic type: an analysis of clinical and prognostic aspects of a rare disease on the basis of cases published in the literature. Archives of gynecology and obstetrics. 2011; 284:1277-82.

5. Witkowski L, Goudie C, Ramos P, Boshari T, Brunet JS, Karnezis AN, Longy M, Knost JA, Saloustros E, McCluggage WG et al. The influence of clinical and genetic factors on patient outcome in small cell carcinoma of the ovary, hypercalcemic type. Gynecologic oncology. 2016; 141:454-60.

6. Harrison ML, Hoskins P, du Bois A, Quinn M, Rustin GJ, Ledermann JA, Baron-Hay S, Friedlander ML. Small cell of the ovary, hypercalcemic type - analysis of combined experience and recommendation for management. A GCIG study. Gynecologic oncology. 2006; 100:233-8.

7. Jamy O, Yaghmour G, Hare F, Martin MG. Population-based Analysis of the Clinical Features of Primary Small Cell Carcinoma of the Ovary. Anticancer research. 2015; 35:3091-5.

8. Karanian-Philippe M, Velasco V, Longy M, Floquet A, Arnould L, Coindre JM, Le Naoures-Méar C, Averous G, Guyon F, MacGrogan G et al. SMARCA4 (BRG1) loss of expression is a useful marker for 
the diagnosis of ovarian small cell carcinoma of the hypercalcemic type (ovarian rhabdoid tumor): a comprehensive analysis of 116 rare gynecologic tumors, 9 soft tissue tumors, and 9 melanomas. The American journal of surgical pathology. 2015; 39:1197-205.

9. Ramos P, Karnezis AN, Craig DW, Sekulic A, Russell ML, Hendricks WP, Corneveaux JJ, Barrett MT, Shumansky K, Yang Y et al. Small cell carcinoma of the ovary, hypercalcemic type, displays frequent inactivating germline and somatic mutations in SMARCA4. Nature genetics. 2014; 46:427-9.

10. Tischkowitz M, Huang S, Banerjee S, Hague J, Hendricks WPD, Huntsman DG, Lang JD, Orlando KA, Oza AM, Pautier P et al. Small-Cell Carcinoma of the Ovary, Hypercalcemic Type-Genetics, New Treatment Targets, and Current Management Guidelines. Clinical cancer research : an official journal of the American Association for Cancer Research. 2020; 26:3908-17.

11. Kim KH, Kim W, Howard TP, Vazquez F, Tsherniak A, Wu JN, Wang W, Haswell JR, Walensky LD, Hahn WC et al. SWI/SNF-mutant cancers depend on catalytic and non-catalytic activity of EZH2. Nature medicine. 2015; 21:1491-6.

12. Wang Y, Chen SY, Karnezis AN, Colborne S, Santos ND, Lang JD, Hendricks WP, Orlando KA, Yap D, Kommoss $\mathrm{F}$ et al. The histone methyltransferase EZH2 is a therapeutic target in small cell carcinoma of the ovary, hypercalcaemic type. The Journal of pathology. 2017; 242:371-83.

13. Chan-Penebre E, Armstrong K, Drew A, Grassian AR, Feldman I, Knutson SK, Kuplast-Barr K, Roche M, Campbell J, Ho P et al. Selective Killing of SMARCA2- and SMARCA4-deficient Small Cell Carcinoma of the Ovary, Hypercalcemic Type Cells by Inhibition of EZH2: In Vitro and In Vivo Preclinical Models. Molecular cancer therapeutics. 2017; 16:850-60.

14. Shorstova T, Marques M, Su J, Johnston J, Kleinman CL, Hamel N, Huang S, Alaoui-Jamali MA, Foulkes WD, Witcher M. SWI/SNF-Compromised Cancers Are Susceptible to Bromodomain Inhibitors. Cancer research. 2019; 79:2761-74.

15. Adeegbe DO, Liu S, Hattersley MM, Bowden M, Zhou CW, Li S, Vlahos R, Grondine M, Dolgalev I, Ivanova EV et al. BET Bromodomain Inhibition Cooperates with PD-1 Blockade to Facilitate Antitumor Response in Kras-Mutant Non-Small Cell Lung Cancer. Cancer immunology research. 2018; 6:1234-45.

16. Wang Y, Chen SY, Colborne S, Lambert G, Shin CY, Santos ND, Orlando KA, Lang JD, Hendricks WPD, Bally MB et al. Histone Deacetylase Inhibitors Synergize with Catalytic Inhibitors of EZH2 to Exhibit Antitumor Activity in Small Cell Carcinoma of the Ovary, Hypercalcemic Type. Molecular cancer therapeutics. 2018; 17:2767-79.

17. Lang JD, Hendricks WPD, Orlando KA, Yin H, Kiefer J, Ramos P, Sharma R, Pirrotte P, Raupach EA, Sereduk C et al. Ponatinib Shows Potent Antitumor Activity in Small Cell Carcinoma of the Ovary Hypercalcemic Type (SCCOHT) through Multikinase Inhibition. Clinical cancer research : an official journal of the American Association for Cancer Research. 2018; 24:1932-43.

18. Wong JP, Todd JR, Finetti MA, McCarthy F, Broncel M, Vyse S, Luczynski MT, Crosier S, Ryall KA, Holmes K et al. Dual Targeting of PDGFRa and FGFR1 Displays Synergistic Efficacy in Malignant Rhabdoid Tumors. Cell reports. 2016; 17:1265-75. 
19. Xue Y, Meehan B, Macdonald E, Venneti S, Wang XQD, Witkowski L, Jelinic P, Kong T, Martinez D, Morin $\mathrm{G}$ et al. CDK4/6 inhibitors target SMARCA4-determined cyclin D1 deficiency in hypercalcemic small cell carcinoma of the ovary. Nature communications. 2019; 10:558.

20. Xue Y, Meehan B, Fu Z, Wang XQD, Fiset PO, Rieker R, Levins C, Kong T, Zhu X, Morin G et al. SMARCA4 loss is synthetic lethal with CDK4/6 inhibition in non-small cell lung cancer. Nature communications. 2019; 10:557.

21. Jelinic P, Ricca J, Van Oudenhove E, Olvera N, Merghoub T, Levine DA, Zamarin D. Immune-Active Microenvironment in Small Cell Carcinoma of the Ovary, Hypercalcemic Type: Rationale for Immune Checkpoint Blockade. Journal of the National Cancer Institute. 2018; 110:787-90.

22. Pejovic T, McCluggage WG, Krieg AJ, Xu F, Lee DM, Witkowski L, Foulkes WD. The dilemma of early preventive oophorectomy in familial small cell carcinoma of the ovary of hypercalcemic type. Gynecologic oncology reports. 2019; 28:47-9.

\section{Figures}
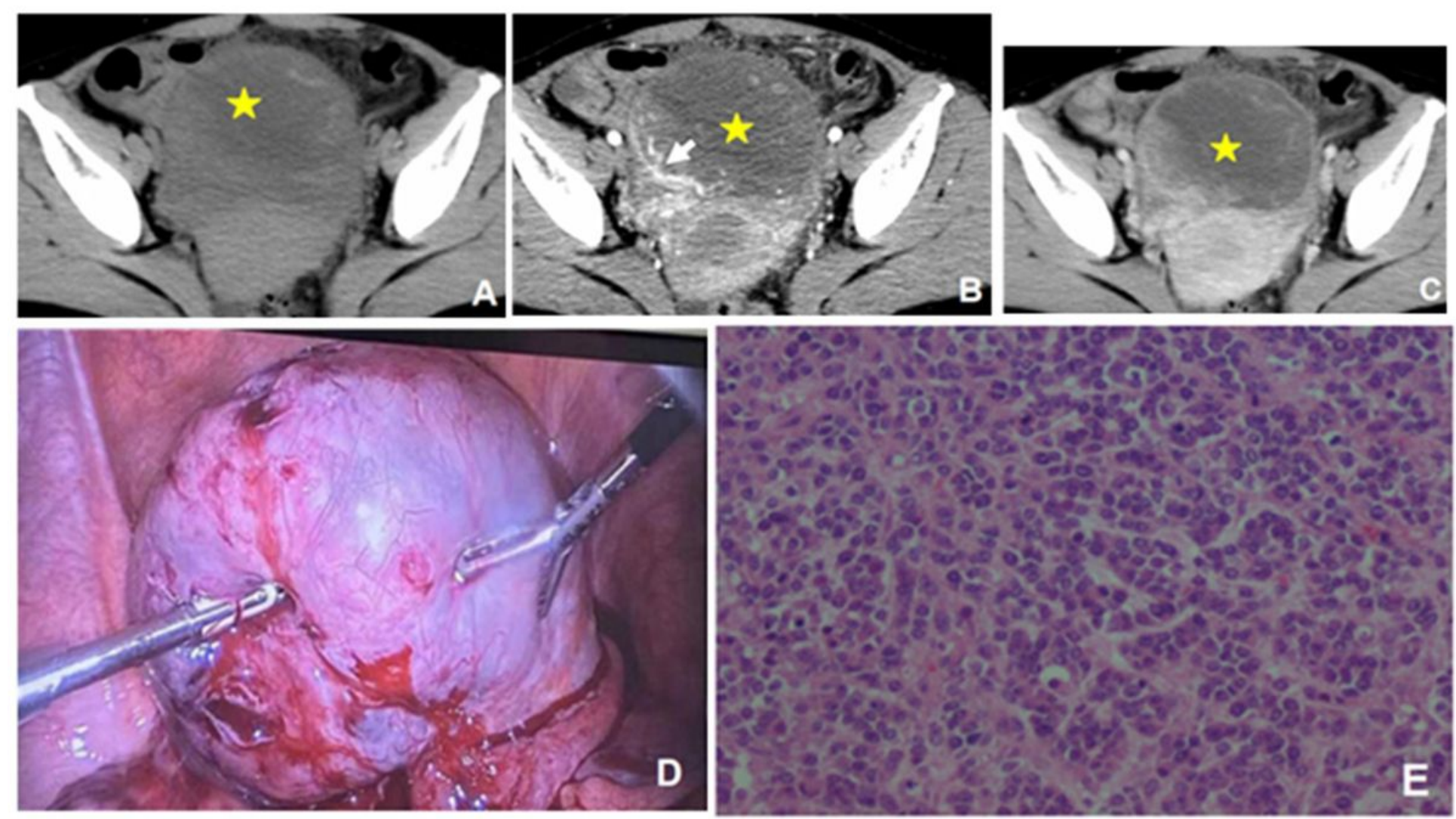

Figure 1

(A-C) Pelvic abdominal CT; (A) a plain scan, (B) an arterial phase of enhancement, and $C$ is a venous phase of enhancement. The density of the mass in the plain scan was not uniform. The lumen was soft tissue density, the extension of the vessels could be seen at the enhancement stage, the solid components of the mass at the venous stage were strengthened, the peripheral enhancement was high, 
suggesting that the mass was malignant. (D) Laparoscopic exploration. The right ovary had a solid but brittle enlargement of $10 \mathrm{~cm}$, a rich blood supply, and a $2 \mathrm{~cm}$ incision. Oozing blood was observed on the surface. (E) Monomorphic round cells with many mitoses $(\times 200)$.
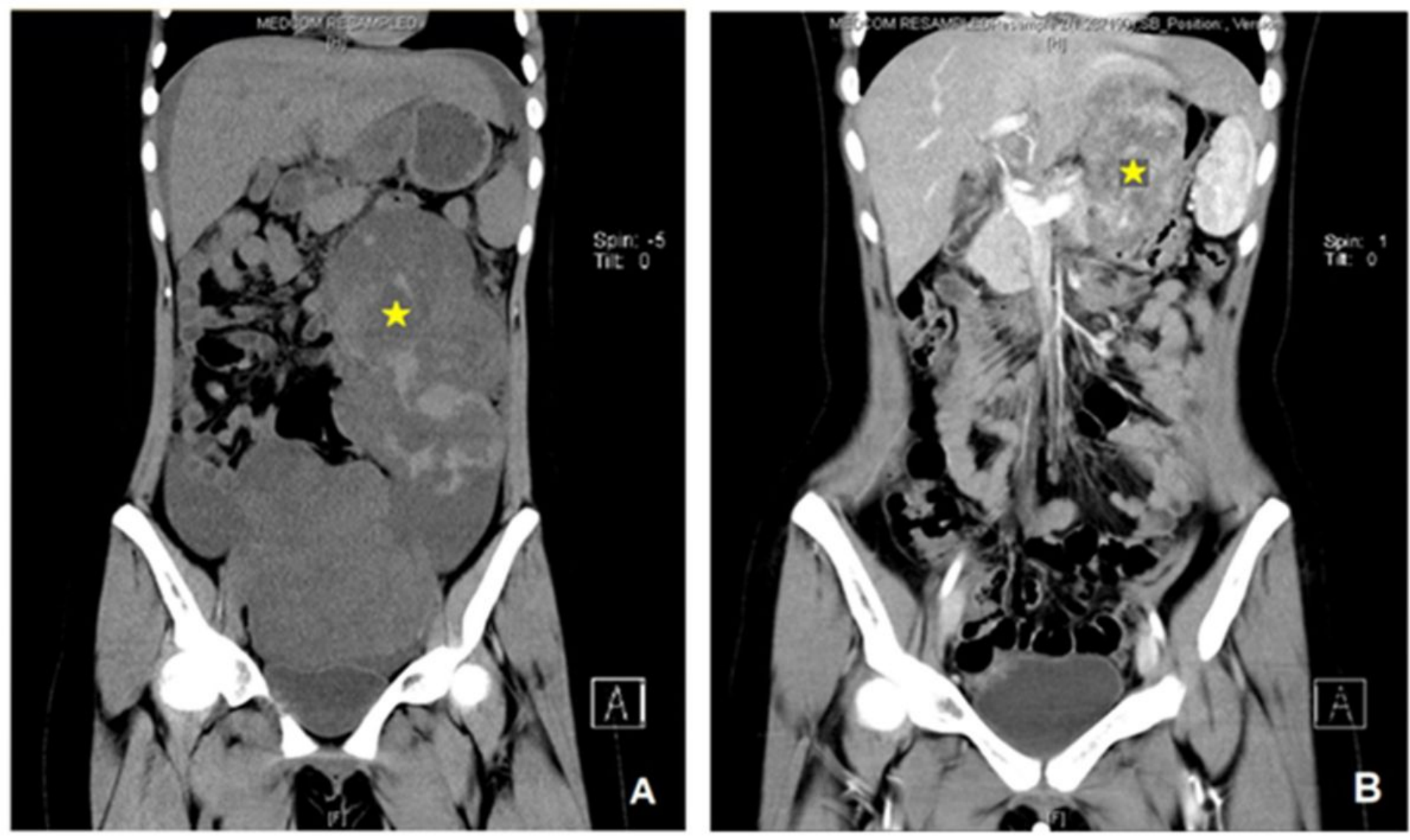

\section{Figure 2}

Pelvic abdominal CT. (A) Huge pelvic mass. (B) A $9 \mathrm{~cm}$ mass between the liver, stomach, and pancreas, increased during the chemotherapy. 

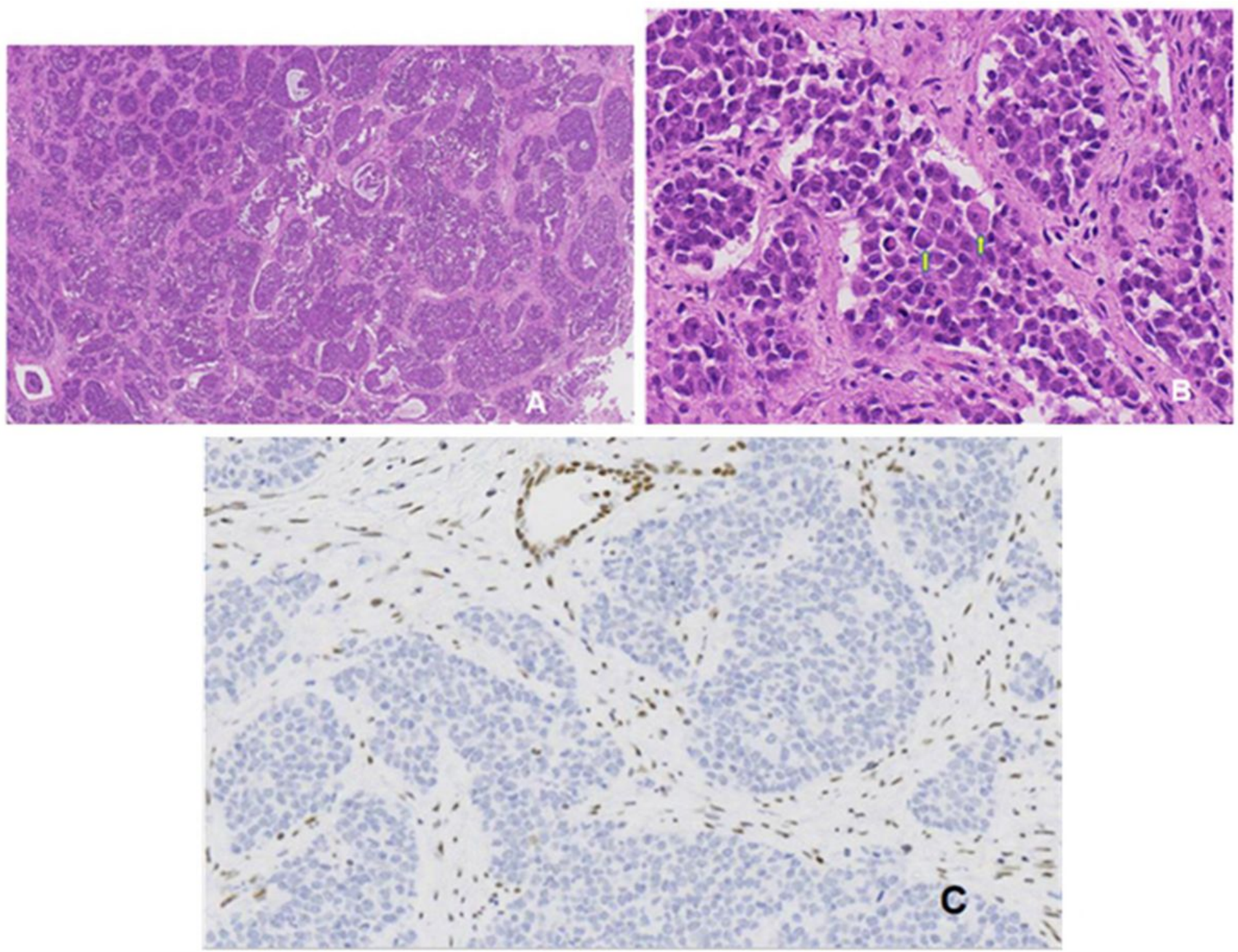

\section{Figure 3}

Pathologic microscopically features of SCCOHT. (A, B) The tumor cells were found to be infiltrating and growing in a flaky, dense nest with small cells, follicle-like structure, and fibrous cords $(\times 40)(A)(\times 200)(B)$. (C) Under the microscope, the tumor cells were BRG-1 negative, while the background cells (endothelial cells) were BRG-1 positive $(\times 200)$. 